\title{
PULMONARY ALVEOLAR MICROLITHIASIS IN PREGNANCY: A RARE CASE REPORT
}

\author{
Hemanta Kumar Sethy ${ }^{1}$, Biswal Pradipta Trilochan ${ }^{2}$, Geetanjali Panda ${ }^{3}$, Milan Misra ${ }^{4}$
}

${ }^{1}$ Associate Professor, Department of Pulmonary Medicine, SCB MCH, Cuttack.

${ }^{2}$ Senior Resident, Department of Pulmonary Medicine, SCB MCH, Cuttack.

${ }^{3}$ Assistant Professor, Department of Pulmonary Medicine, SCB MCH, Cuttack.

${ }^{4}$ Post Graduate, Department of Pulmonary Medicine, SCB MCH, Cuttack.

\section{ABSTRACT}

Pulmonary Alveolar Microlithiasis (PAM) is a very rare diffuse lung disease, affecting both lungs by intra-alveolar deposition of calcium phosphates in form of microliths. There have been about 700 cases of PAM recorded in the medical literature. The etiopathogenesis of the disease is unknown. It is now thought to be an autosomal recessive disease caused by deletion of $\mathrm{Na}-\mathrm{Pi}$ cotransporter (Npt2b) in alveolar epithelial cells due to defective DNA mutation in the gene SLC34A2. The disease is slowly progressive, usually detected in $3^{\text {rd }} / 4^{\text {th }}$ decades of life and death is due to cardiorespiratory failure. The patients detected with PAM in pregnancy are exceptional. Very few cases were reported till date. We report a pregnant lady presenting with PAM delivering vaginally a healthy female child in spite of cardiomegally and severe PAH.

KEYWORDS: PAM, Calcification, Familial, SLC34A2, Pregnancy, Cor Pulmonale.

HOW TO CITE THIS ARTICLE: Hemanta Kumar Sethy, Biswal Pradipta Trilochan, Geetanjali Panda, Milan Misra. "Pulmonary Alveolar Microlithiasis in Pregnancy: A Rare Case Report." Journal of Evolution of Medical and Dental Sciences 2015; Vol. 4, Issue 98, December 07; Page: 16387-16389, DOI: 10.14260/jemds/2015/2424

\section{INTRODUCTION}

PAM is characterized by intra-alveolar deposition of calcium phosphates in both lungs in form of microliths ranging from 0.02 to $0.3 \mathrm{~cm}$ in size.[1] in absence of a known calcium metabolic disorder.[2] The disease was first isolated by Friedrich during an autopsy in 1856, first reported by Harbitz in 1918, first radiologically described by Schildknecht in 1932 and Puhr first named the disease in 1933. It occurs in familial and sporadic forms. The familial cases are known to be transmitted in an autosomal recessive way with 100\% penetrance.[2] Around one-third of cases are found to be hereditary.[2] Familial cases predominantly affect females, whereas sporadic cases are usually seen in males.[3] The etiopathogenesis is unclear. The present implication for intraalveolar microlith deposition is: Na Pi co-transporter (Npt2b) deletion following defective mutation in the gene "SLC34A2" (In short arm of chromosome 4) in the apical portions of alveolar type II cells. $\left.{ }^{4}, 5\right]$ Another concept is "The increased and condensated alveolar mucous membranes" due to declining mucocilliary function leads to microlith formation.[6]

It has been suggested that the alveolar wall in affected subjects tends to be alkaline due to an abnormality of carbonic anhydrase that favours calcium deposits.[1] The microliths gradually grow within the alveoli to fill the entire alveolar space and damage the alveolar walls with fibrous tissue replacement. [2] But $50 \%$ of patients present with minimal or no symptoms in spite of maximal lung involvement bilaterally. More often the disease is detected incidentally by CXR during routine examinations.[2] The patients present with dyspnea, dry cough, rarely scanty mucoid expectoration with microliths or hemoptysis and on examination inspiratory crackles, sometimes wheezes or features of cor pulmonale.

Death occurs 10-15 yrs. following the diagnosis.[6] We detected the case after delivery with history of symptoms in

Financial or Other, Competing Interest: None.

Submission 20-11-2015, Peer Review 21-11-2015,

Acceptance 30-11-2015, Published 07-12-2015.

Corresponding Author:

Dr. Biswal Pradipta Trilochan,

Senior Resident,

Department of Pulmonary Medicine,

SCB MCH, Cuttack.

E-mail: drpradipta.biswal1@gmail.com

DOI:10.14260/jemds/2015/2424 $2^{\text {nd }}$ trimester of pregnancy.

\section{CASE REPORT}

A 27 yrs. old female admitted to Pulmonary Medicine, SCB Medical College, Cuttack 1 week after delivery in Obstetric ward with history of progressive dyspnea, dry cough and swelling of both legs of 3 months' duration. She had no previous history of any chronic respiratory illness, rheumatological disorders, hypertension, DM, HIV or any prolonged antibiotics/immunosuppressive medications. Family history was not suggestive of any known genetic disease among cousins/siblings. On admission, physical examination revealed prominent clubbing, Sp02 76\%, tachycardia, tachypnea, BP $110 / 80 \mathrm{mmHg}$, raised JVP, bilateral pedal oedema, tubular bronchial breath sounds over right infrascapular region and late inspiratory crackles, predominantly over bilateral infrascapular regions. Haematological parameters displayed leucocytosis (TLC $18,800, \mathrm{~N} 72 \%$ ), TPC normal, PCV 27\%, Hb 7.8\%, microcytic hypochromic anemia, but serological parameters showed hypokalemia, hypoproteinemia, hypoalbuminemia, hyperprolactinemia $(51.31 \mathrm{ng} / \mathrm{ml})$, but serum total/ionized calcium, phosphorous, sodium, creatinine, urea and liver profile all within normal range. Urine report revealed albuminuria.

CXR PA view showed diffuse symmetric dense bilateral micronodular opacities involving all zones with increased density towards bases. HRCT thorax revealed numerous sand like calcifications throughout bilateral lung fields with middle to lower zone predilection, calcified interlobular septa, sand like calcification in peribronchial and subpleural distribution (Peripheral, Mediastinal, Fissural, Pericardiac) suggesting PAM with cardiomegally, pericardial effusion and dilated pulmonary trunk. Echocardiography demonstrated dilated RA and RV, severe PAH, TR, moderate AR with moderate pericardial effusion, but normal LV systolic function. Spirometry showed mild restriction and DLCO was $48 \%$ predicted. Ultrasonography revealed distended IVC and mild ascites. Bronchoscopy showed no visible intraluminal abnormalities and Transbronchial Lung Biopsy (TBLB) not done because of severe PAH. BAL fluid showed benign squamous epithelial cells, plenty pus cells on gram stain and no AFB on ZN staining, but pseudomonas on aerobic culture. 
The patient after anti-pseudomonal antibiotics, diuretics and dihydrophosphonate was discharged on request and found to be doing well.

\section{DISCUSSION}

Intra-alveolar deposition of calcified concretions in both lungs in absence of a known calcium metabolic disorder is very rare and detection of PAM in pregnancy is still rare. There have been about 700 cases of PAM recorded in the medical literature.[7] The slow and progressive deposition of calcium phosphates keep the patients asymptomatic for a prolonged period. The disease starts early in childhood, but clinical symptoms arise much later in $3^{\text {rd }} / 4^{\text {th }}$ decades of life. The pregnant patients with PAM usually become symptomatic in $2^{\text {nd }}$ to $3^{\text {rd }}$ trimester of gestation. Our case became symptomatic in $2^{\text {nd }}$ trimester of pregnancy. Digital clubbing was a prominent feature posing pulmonary fibrosis, Meadow's syndrome, Eisenmenger's syndrome as a part of discussion. Continuous deposition of calcium as small stones can inflict injury, inflammation and fibrosis that may lead to clubbing. Presence of bronchial breath sound as a sign of consolidation found to be persisted even after antipseudomonal antibiotics.

"Sand storm" appearance (Diffusely scattered bilateral areas of micronodular calcification in middle and lower zones with silhouetted heart border and diaphragm) on CXR is a classical pathognomonic diagnostic feature of PAM.[8] CT confirms relatively symmetrical abnormalities and differentiates parenchymal septal and subpleural (Peripheral, Mediastinal, Fissural) nodular calcifications more distinctly and demostrates each lobe to be surrounded by a fine dense outline like a 'stony lung.' Yesner suggested that there are two types of alveolar microlithiasis. "One type is characterized by round or oval microliths that may have a genetic basis and the other by irregular bosselated microliths that are largely due to mitral stenosis." [9] Confirmation with CT scan, scintigraphy, transbronchial biopsy can be done. Presence of sand storm appearance and round/oval calcifications in our case form the basis of diagnosis of PAM. Lung biopsy is always confirmatory and demonstrates laminated concentric layers of calcium phosphates/carbonates of size 0.02 to $0.3 \mathrm{~cm}$ without cells.[1] Occasionally, microliths can be found in sputum or BAL which may contain alveolar macrophages with or without carbon particles and in our case microliths were not found in above specimens.

Very few reported cases with PAM in pregnancy are collected in world literatures till now. We found our patient to become symptomatic around $2^{\text {nd }}$ trimester. In such cases, the child is usually delivered prematurely by Caesarean section. ${ }^{[3]}$ and in some cases full term delivery has been reported. It should be considered that this disease with no effective treatment may rarely progress into end-stage pulmonary disease due to conditions which alter pulmonary functions, such as pregnancy. But rapid progression of the disease is possible. The role of PAM in pregnancy is still unknown. The presence of hypocalcemia in different trimesters. ${ }^{[10]}$ whether responsible in amplifying abnormal deposition of demineralised bone calcium in soft tissues like lungs due to improper signals following impairment of normal functioning of muscles, nerves and vesssels is debatable. In our case, the mother delivered a full term baby normally, vaginally. There was typical evolution of PAM in late stages and fetal development was normal. It seems that PAM is not an indication for abortion or premature interruption of pregnancy even in the late stages.

Currently, the only effective therapy is lung transplantation. Disodium etidronate, a diphosphonate has been used with mixed results. It acts by inhibiting the formation of new pulmonary calcium-phosphate crystallization and resolving previously formed calcifications as observed in other diseases. Whole-lung EDTA Washing can reduce the burden of stones in the lungs and a low-phosphate diet prevents stone formation. ${ }^{[5]}$ Gene insertion by viral vectors is under trial.

\section{ABBREVIATIONS}

CXR- Chest X-ray, DM- Diabetes Mellitus, HIV- Human Immunodeficiency Virus, Sp02- Oxygen saturation, TLC- Total Leukocyte Count, TPC- Total Platelet Count, PCV- Packed Cell Volume, HRCT- High Resolution Computed Tomography, RARight Atrium, RV- Right Ventricle, LV- Left Ventricle, TRTricuspid Regurgitation, AR-Aortic Regurgitation, PAHPulmonary Arterial Hypertension, IVC- Inferior Venacava, BAL- Bronchoalveolar Lavage, AFB- Acid Fast Bacillus, ZN Ziehl Neelsen.

\section{REFERENCES}

1. Fabian Rodri Guez, Jorge Ferrer, Leonel Briozzo, \& Jose Pons. Pulmonary alveolar microlithiasis and pregnancy. The Journal of Maternal-Fetal and Neonatal Medicine, April 2006;19(4):239-241.

2. Mariotta, A Ricci, M Papale, F De Clementi, B Sposato, L Guidi, et al. Pulmonary alveolar microlithiasis: report on 576 cases published in the literature. Sarcoidosis Vasc Diffuse Lung Dis 21(3) (2004), pp. 173-181.

3. Gülnur Erdem, Aslı Göktan, Fatih Erbay, Tamer Baysal. Pulmonary Alveolar Microlithiasis and Preterm Delivery: A Case Report. Turk Toraks Derg 2014;15:33-5.

4. M Traebert, O Hattenhauer, H Murer, B Kaissling, J Biber. Expression of type II Na-P (i) co-transporter in alveolar type II cells. Am J Physiol, 277 (5 Pt 1) (1999), pp. L868-L873.

5. Atsushi Saito, Nikolaos M. Nikolaidis, Hassane Amlal, Yasuaki Uehara, et al. Modelling pulmonary alveolar microlithiasis by epithelial deletion of the Npt2b sodium phosphate cotransporter reveals putative biomarkers and strategies for treatment. Science Translational Medicine, 11 Nov 2015: Vol. 7, Issue 313, pp. 313ra181D0I: 10.1126/scitranslmed.aac8577.

6. VM Lauta. Pulmonary alveolar microlithiasis: an overview of clinical and pathological features together with possible therapies. Respir Med, 97 (10) (2003), pp. 1081-1085.

7. PUBLIC RELEASE: 15-APR-2015. UC researcher receives grant of almost $\$ 1.6$ million to study rare lung disease. UNIVERSITY OF CINCINNATI ACADEMIC HEALTH CENTER.

8. Gasparetto EL, Tazoniero P, Escuissato DL, Marchiori E, Frare E, Silva RL, et al. (2004) Pulmonary alveolar microlithiasis presenting with crazy-paving pattern on high resolution CT. Br J Radiol 77:974-976.

9. Yesner R. Pulmonary alveolar microlithiasis revisited (Correspondence). N Engl J Med 2003;348:84-85.

10. Pitkin RM, Gebhard MP. Serum calcium concentrations in human pregnancy. Am J Obstet Gynecol 1977 Apr 1;127(7):775-8. 


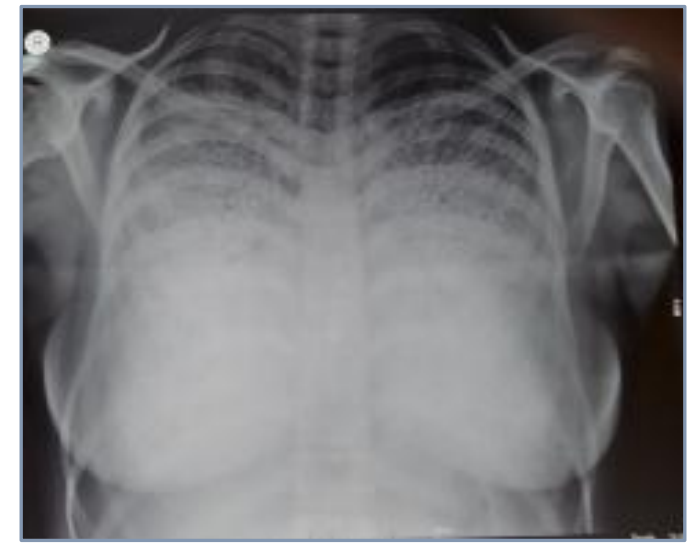

Fig. 1: Chest X-ray PA view: Scattered Diffuse Nodular Infiltrations showing "Sand Storm" Appearance

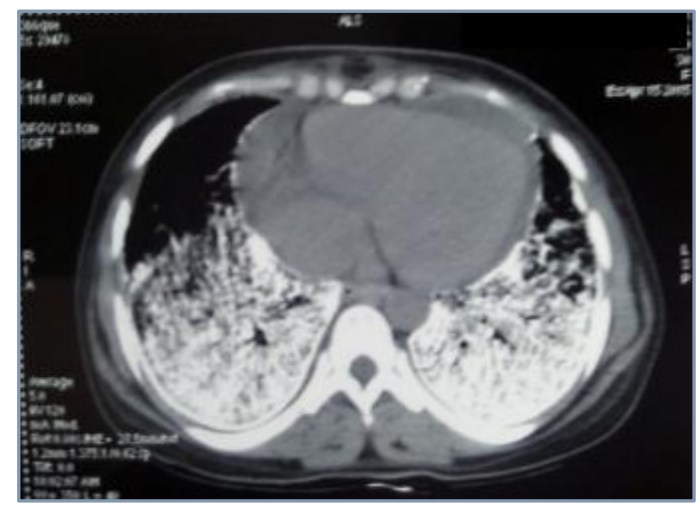

Fig. 2: HRCT Thorax (Axial)

Conglomerated Parenchymal Nodular Calcifications Forming Symmetrical Consolidations with Subpleural Calcifications in Both Lower Lobes and Subpericardial Nodular Calcifications

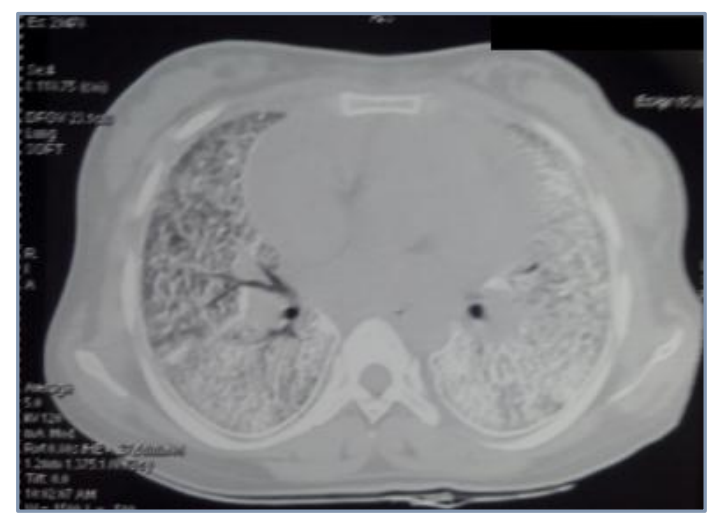

Fig. 3: CT Thorax (Axial- Bone Window) Parenchymal and Subpleural Nodular Calcifications showing Bone Density and Cardiomegaly

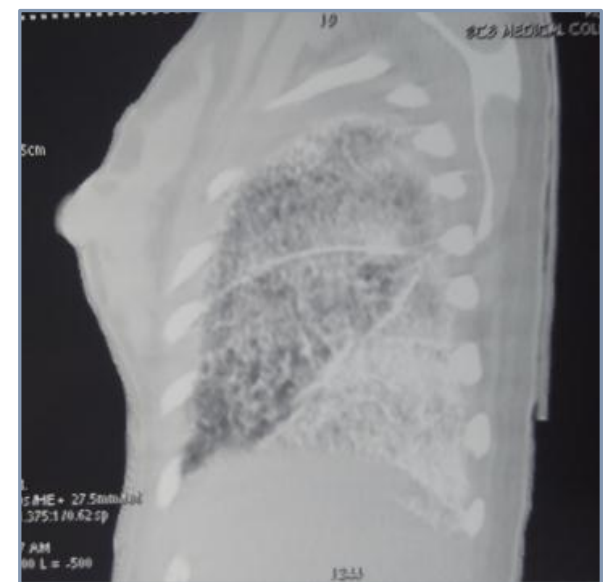

Fig. 4: CT Thorax (Sagittal- Bone Window) Interstitial Septal, Interlobar, Parenchymal Nodular Calcifications of Right Lung

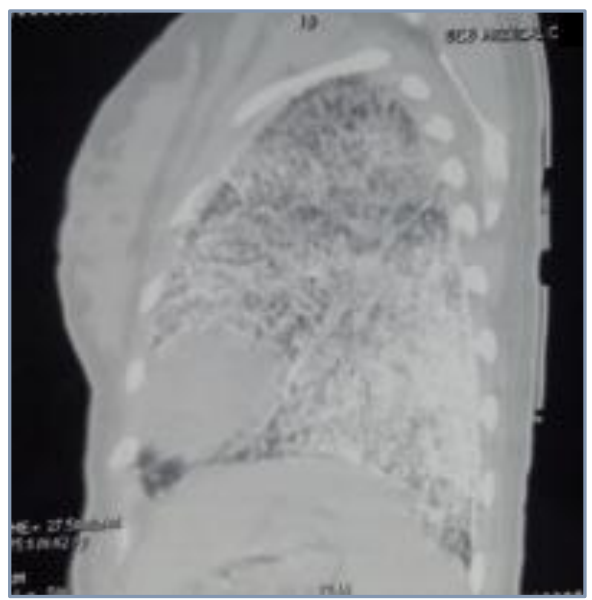

Fig. 5: CT Thorax (Sagittal- Bone Window) Interstitial Septal, Interlobar, Peripheral, Parenchymal Nodular Calcifications of Left Lung

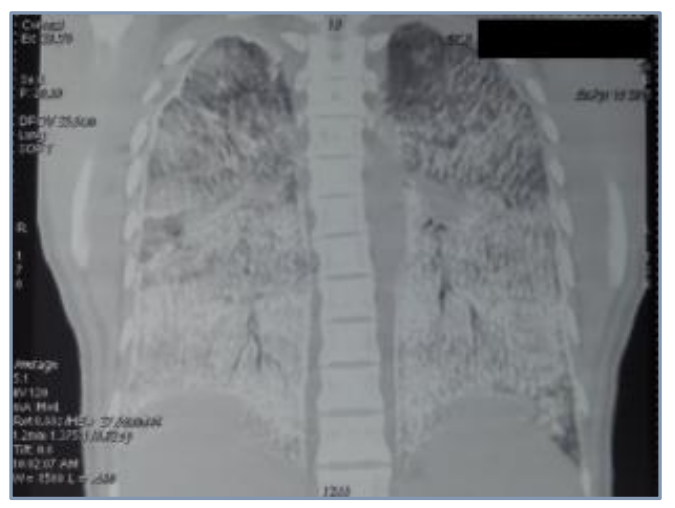

Fig. 6: CT Thorax (Coronal- Bone Window) Parenchymal, Interstitial, Subpleural (Peripheral, Mediastinal, Diaphragmatic, Interlobar) Calcifications with Air Bronchogram and "Sand Storm" Appearance giving rise to "Stony Lung" 